\title{
Schlieren imaging of spatial magnetic fields by hollow-cone illumination
}

\author{
Ken Harada ${ }^{1}$, Hiroshi Nakajima ${ }^{2}$, Shigeo Mori $^{2}$ and Yoshio Takahashi ${ }^{3}$ \\ ${ }^{1}$ RIKEN, Hatoyama, Saitama, Japan, ${ }^{2}$ Department of Materials Science, Osaka Prefecture University, Sakai, \\ Osaka, Japan, ${ }^{3}$ Research \& Development Group, Hitachi, Ltd., Hatoyama, Saitama, Japan
}

We have been using the recently-developed hollow-cone Foucault imaging method [1, 2] for studying magnetization structures, such as magnetic domains and domain walls. The hollow-cone Foucault imaging has two modes depending on the inclination angle of the illumination beam, bright-field mode and dark-field mode. We have also found a specially controlled mode, a schlieren imaging mode, between these Foucault modes. Although the schlieren imaging is a method developed in the middle of the 19th century to qualitatively visualize distributions of refractive indices of transparent media, such as air, it is still used today as a method for visualizing sound shock waves generated by high-speed flying objects [3]. In this study, we have extended the schlieren imaging method to be used in transmission electron microscopes (TEMs) to directly observe magnetic fields around specimens by using the hollow-cone illumination. The specimens were Fe0.88Ga0.12 alloys (at\%) having large magnetostriction at room temperature [4]. Their thin films (250 $\mathrm{nm}$ in thickness) were prepared by a focused ion beam instrument (NB-5000). The visualization experiments were carried out by using a 200-kV thermal field-emission TEM (JEM-2100F). Figure 1 (a) shows a schematic diagram of the optical system for the hollow-cone illumination imaging. The parallel electron beams having less than a 10-6 rad diffusion angle are irradiated on the specimen with the inclination angles in $\mathrm{X}$ and $\mathrm{Y}$ directions controlled by using the beam deflector system placed above the specimen. The circulating electron beam is illuminated in all azimuthal directions around the optical axis. Figures 1(b), (c), and (d) show schematic relation between the selected area (SA) aperture and diffraction spots of small-angle hollow-cone beams. The 0-th transmission center spots indicated in red in Figs. 1(b), (c), and (d) clarify the difference of three modes of the hollow-cone imaging, bright-field hollow-cone Foucault mode in (b), hollow-cone schlieren mode in (c), and dark-field hollow-cone Foucault mode in (d). Figures 1(e), (f), and (g) show the images in these modes, respectively. Magnetic domains and domain walls are clearly shown in the hollow-cone Foucault images in (e) and (g), while the schlieren image in (f) has no magnetization-related contrast inside the thin film. When we manually tuned the display intensity in the vacuum area outside the specimen, we were able to obtain various image contrasts in the schlieren imaging between bright-field and dark-field hollow-cone Foucault imaging. Figure 2 shows series of schlieren images due to successive changes in the inclination beam angles by $2.0 \times 10-5 \mathrm{rad}$ at each step. The positions of large changes in contrast modulations coincided with the places where the magnetic domain walls terminated at specimen edges. The contrast can be interpreted as magnetic fields leaking out to the vacuum area from the specimen. These experimental results are still at the trial level and therefore are qualitative. We are working on further developments of the hollow-cone schlieren imaging method to obtain quantitative data for determining magnetic field behavior. 


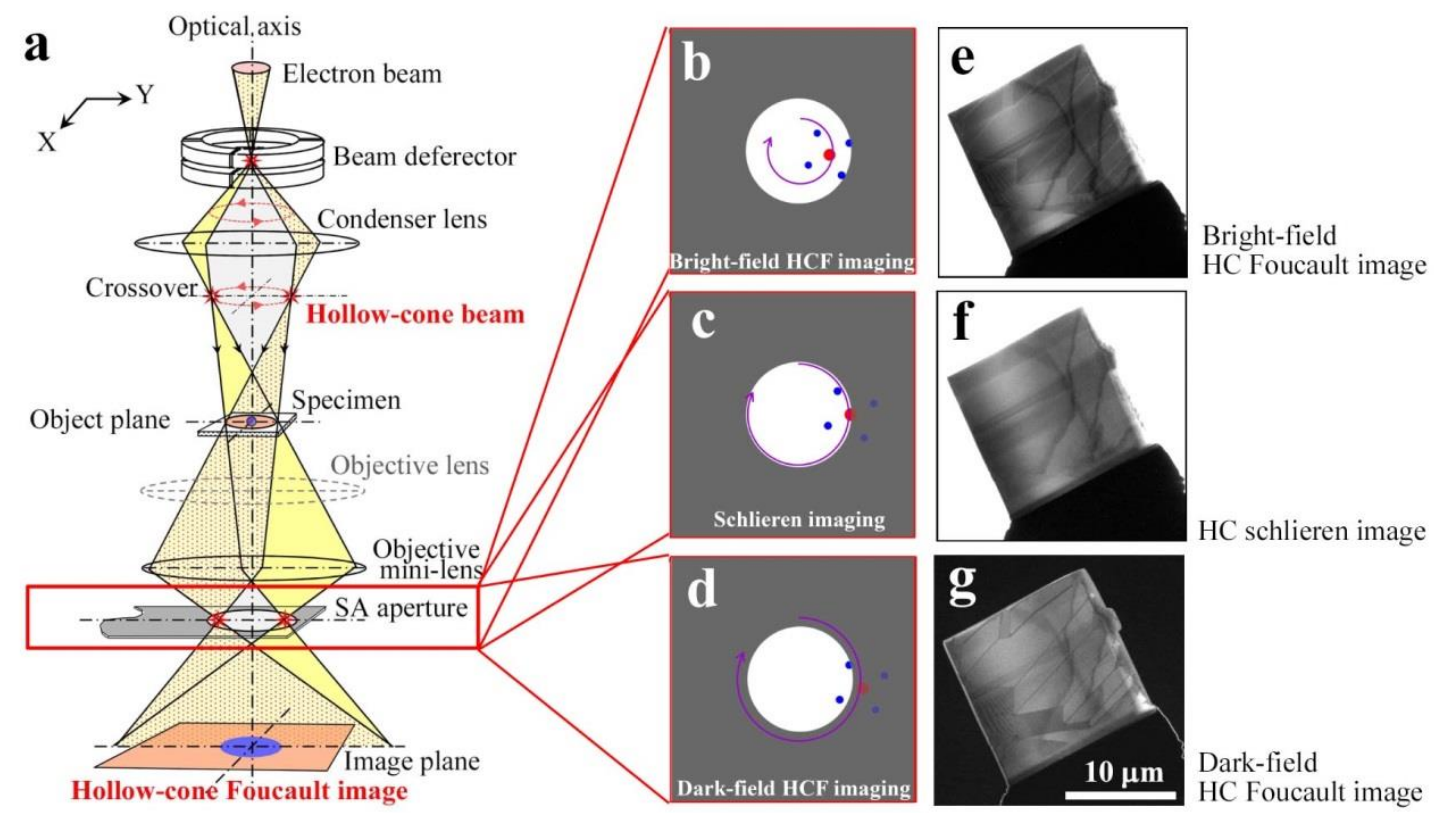

Figure 1. (a) Schematic diagram of the optical system for the hollow-cone Foucault (HCF) imaging, (b) schematics of the relation between an SA aperture hole and diffraction spots for bright-field HCF imaging, (c) for hollow-cone (HC) schlieren imaging, (d) for dark field HCF imaging, (e) bright-field HCF image, (f) HC schlieren image, and (g) dark-field HCF image.(a) Schematic diagram of the optical system for the hollowcone Foucault (HCF) imaging, (b) schematics of the relation between an SA aperture hole and diffraction spots for bright-field HCF imaging, (c) for hollow-cone (HC) schlieren imaging, (d) for dark-field HCF imaging, (e) bright-field $\mathrm{HCF}$ image, (f) HC schlieren image, and (g) dark-field HCF image.

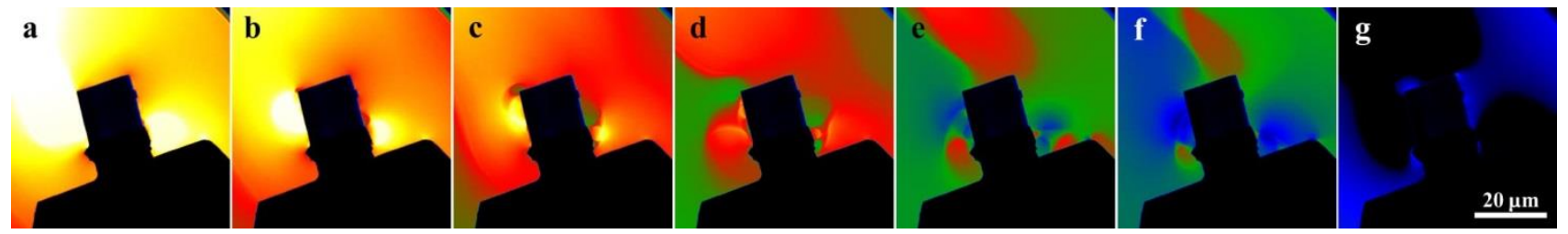

Figure 2. Series of schlieren images depending on the inclination beam angle of the illumination beam, (a) inclination beam angle of 3.0×10-4 rad, (b) $3.2 \times 10-4 \mathrm{rad,} \mathrm{(c)} 3.4 \times 10-4 \mathrm{rad}$, (d) $3.6 \times 10-4 \mathrm{rad}$, (e) $3.8 \times 10-4 \mathrm{rad}$, (f) $4.0 \times 10-4 \mathrm{rad}$, and (g) $4.2 \times 10-4 \mathrm{rad}$.

\section{References}

[1] K. Harada et al., Appl. Phys. Exp., 12 (2019) 042003.

[2] K. Harada et al., Microsc. Microanal., 25 (2019) 120.

[3] https://www.nasa.gov/centers/armstrong/features/supersonic-shockwave-interaction.html

[4] H. D. Chopra and M. Wutig, Nature, 521 (2015) 340.

[5] The authors thank Dr. Y. A. Ono of RIKEN for his valuable discussion, Prof. H. Numakura of Osaka Prefecture University for providing us $\mathrm{Fe}_{0.88} \mathrm{Ga}_{0.12}$ alloys, Ms. K. Shimada of RIKEN for preparing thin specimens, and Mr. A. Kawaguchi, Dr. A. Kotani, Mr. Y. Fujibayashi, Mr. K. Uchihashi of Osaka Prefecture University for their technical supports. This work was supported by KAKENHI, Grant-in-Aid for Scientific Research ((B) 18H03475 and 20K20555). 